isopropylphosphoryl enzyme, as found by Wilson for other cationic compounds.

The combination of intrinsic activity and 'promotion' will account for many of the variations in activity observed in hydroxylamine derivatives. Thus nicotinhydroxamic acid methiodide, which shows the 'promotion' effect, is a superior reactivator of the diethylphosphoryl enzyme to nicotin. hydroxamic acid (Wilson, Ginsburg \& Meislich, 1955), although the latter will react about three times as rapidly with simple organophosphorus compounds at $\mathrm{pH} \mathbf{7 \cdot 4}$. Pyridine-2-aldoxime methiodide possesses both high reactivity and the ability to form strong complexes and is accordingly very effective as a reactivating agent.

The intrinsic activity of any reactivator will depend both on the ability of the reactivating agent to react directly with organophosphorus compounds and on the orientation and position of the reactivator in the complex. This latter factor may in some instances be critical and may account for the unexpectedly low reactivating power of pyridine-3aldoxime methiodide.

The way in which the reactivator fits on to the enzyme surface will depend both on the geometry of the reactivator and inhibited enzyme and on the attraction between the cationic centre in the reactivator and the anionic site on the enzyme. Since the values of $k_{3}$ for reactivation of $\mathrm{ChE}$ inhibited with TEPP by pyridine-2- and -4-aldoxime methiodides differ by a factor of only about 2 , and since the reactivities of these oximes with organophosphorus compounds are similar (see Table 1), it seems probable that the two oximes are orientated in a similar manner on the enzyme surface. If this is so, the quaternary nitrogen atom in the 4-isomer may be appreciably further from the anionic site on the enzyme surface than is the quaternary nitrogen atom in the 2 -isomer, resulting in weaker binding and a much higher value for $K$ as found experimentally.

Pyridine-2-aldoxime methiodide and isonitroso- acetone are about equally reactive with organo-

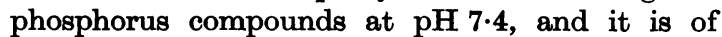
interest that the $k_{3}$ values also differ only by a factor of about 2, which is consistent with the complexes formed by both compounds being suitably orientated to facilitate reactivation. For ChE inhibited with Sarin, $k_{3}$ for pyridine-4-aldoxime methiodide is considerably less than that for isonitrosoacetone, which would suggest that the pyridine compound is forced by the strong binding into a less satisfactory orientation for reactivation to occur.

\section{SUMMARY}

1. Kinetic studies of reactivation by pyridine-2and -4-aldoxime methiodides of erythrocyte cholinesterase inhibited with organophosphorus compounds indicate that a preliminary step is the formation of a complex between the inhibited enzyme and the reactivator.

2. The reactivating powers of the isomeric pyridinealdoxime methiodides are compared in terms of their intrinsic reactivities with organophosphorus inhibitors, the strength of the complexes and the orientation of the reactivator in the complex.

Acknowledgement is made to the Controller of H.M. Stationery Office for permission to publish this paper.

\section{REFERENCES}

Bernhard, S. A. (1955). J. Amer. chem. Soc. 77, 1973.

Davies, D. R. \& Green, A. L. (1955). Disc. Faraday Soc. 20, 269.

Davies, D. R. \& Green, A. L. (1956). Biochem. J. 63, 529.

Dostrovsky, I. \& Halmann, M. (1953). J. chem. Soc. p. 502.

Eadie, G. S. (1942). J. biol. Chem. 146, 85.

Green, A. L. \& Saville, B. (1956). J. chem. Soc. p. 3887.

Green, A. L. \& Smith, H. J. (1958). Biochem. J. 68, 28.

Wilson, I. B. (1955). Disc. Faraday Soc. 20, 119.

Wilson, I. B. \& Ginsburg, S. (1955). Biochim. biophys. Acta, 18, 168.

Wilson, I. B., Ginsburg, S. \& Meislich, E. K. (1955). J. Amer. chem. Soc. 77, 4286.

\title{
The $\boldsymbol{N}$-Terminal Groups of Calf-Thymus Histones
}

\author{
By D. M. P. PHILLIPS \\ Chester Beatty Research Institute, Institute of Cancer Research, Royal Cancer Hospital, London, S.W. 3
}

(Received 14 May 1957)

It is well established that the histones of calfthymus nucleoprotein consist of a mixture of proteins, and partial fractionations of them have been achieved (e.g. Stedman \& Stedman, 1951; Davison \& Butler, 1954; Daly \& Mirsky, 1955; Crampton, Moore \& Stein, 1955). As a continuation of the work in this Laboratory, the study of the
$N$-terminal groups of the mixed histones from calfthymus glands was undertaken by the fluorodinitrobenzene method (Sanger, 1945). It was expected that the results would be helpful in controlling the fractionation of the histones. While this work was in progress a dissertation on this topic appeared (Haley, 1955) and the work described has since been 
published as part of a wider study of histones (Luck et al. 1956). However, the results obtained here are not in good agreement with those reported.

\section{MATERIALS AND METHODS}

Preparation of histones. The mixed histones were prepared from previously isolated nucleoprotein from fresh frozen calf-thymus glands by the method of Davison, James, Shooter \& Butler (1954). The series of preparations used were labelled $\mathrm{H} 1, \mathrm{H} 16, \mathrm{H} 17$ and $\mathrm{H} 18$. Some fractions of the histones were also examined. Thus the sodium chloride-ammonia treatment of the histones $\mathrm{H} 18$ by the method of Davison \& Shooter (1956) gave a histone precipitate (H 18 A) and soluble histones in the supernatant (H 18B). The citric acid extraction of whole-thymus nucleoprotein (Davison \& Butler, 1954) yielded a small amount of histone (labelled $\mathrm{H} 7$ ), which was then separated into two fractions, $H 7 / 1$ and $H 7 / 2$, by column chromatography on carboxymethylcellulose by the method of Davison \& Shooter (1956). In addition the whole of the proteins extracted by acid from thymus glands have been examined. For this purpose, thymus gland $(50 \mathrm{~g}$.) was blended in $0.25 \mathrm{~N}-\mathrm{HCl}$ at room temperature, and the suspension centrifuged to remove large insoluble debris. The supernatant suspension was then dialysed until free of $\mathrm{Cl}^{-}$ ions and centrifuged in a Model $L$ Spinco ultracentrifuge at $24000 \mathrm{~g}$ (av.) for $1 \mathrm{hr}$. The clear supernatant was then freeze-dried (yield $2.0 \mathrm{~g}$.) and examined for its $N$-terminal groups (preparation $\mathrm{H}$ 19).

Reaction with 1-fluoro-2:4-dinitrobenzene. A variety of conditions of coupling with 1-fluoro-2:4-dinitrobenzene (FDNB) have been tried, including an ethanol-bicarbonate medium (Sanger, 1945). In general, higher yields of total end groups were obtained in a guanidine hydrochloridepotassium bicarbonate medium, the purpose of which was to break up aggregates. A typical preparation is given below. The histone hydrochloride was dissolved in $6 \mathrm{M}$ guanidine hydrochloride to give a concentration of 10$20 \mathrm{mg}$. $/ \mathrm{ml}$. Solid $\mathrm{KHCO}_{3}$ was added to a concentration of $10-15 \mathrm{mg}$. $/ \mathrm{ml}$., and FDNB to $0.05-0.1 \mathrm{ml} . / \mathrm{ml}$. The mixture was shaken at about $20^{\circ}$ for $6-24 \mathrm{hr}$. It was then acidified, diluted with 3 vol. of water and extracted once with ether, the layers being separated by centrifuging. The yellow precipitate was washed repeatedly with $\mathrm{N}-\mathrm{HCl}$ and centrifuged (this did not clarify the supernatant if $0.1 \mathrm{~N}-\mathrm{HCl}$ or water was used). Final traces of reagent and dinitrophenol were removed by three acetone washes and an ether wash.

The weight of dinitrophenylhistones (DNP-histones) obtained was $110-120 \%$ of the weight of histones taken. The DNP-histones were then hydrolysed, generally for $4.5 \mathrm{hr}$., in $11.1 \mathrm{~N}-\mathrm{HCl}$ at $100^{\circ}$ (Porter \& Sanger, 1948), since DNPproline was found to be an end group. The completeness of liberation of the more stable derivatives, such as DNP. valine, was checked by hydrolyses in $6 \mathrm{~N}-\mathrm{HCl}$ at $110^{\circ}$ or $120^{\circ}$. Hydrolyses were done in sealed tubes, with $1.5 \mathrm{ml}$. of acid/ $20 \mathrm{mg}$. of DNP-histones, this being a convenient weight for one complete analysis. The hydrolysates were diluted with water and extracted four times with ether in glass-stoppered test tubes. Each ether extract was washed with $0 \cdot 1 \mathrm{~N}-\mathrm{HCl}$ to remove any entrained acid-soluble materials such as $\epsilon$-DNP-lysine. The ether used in this work was freed from peroxide by passing through a column of activated alumina (Dasler \& Bauer, 1946).
The ether-soluble materials, after sublimation of excess of dinitrophenol (Mills, 1952), were transferred in three lots of acetone to a two-dimensional chromatogram on no. 7 Whatman filter paper buffered with $0.05 \mathrm{M}$-phthalate buffer (pH 6). This was then run in the dark for $30-40 \mathrm{hr}$. with 2-methylbutan-2-ol saturated with the phthalate buffer (Blackburn \& Lowther, 1951), dried at room temperature and run in the other dimension overnight with $1.5 \mathrm{M}$-sodium phosphate buffer (pH 6) (Levy, 1954), $22 \mathrm{ml}$./chromatogram being used. The papers were dried at room temperature in the dark and the spots eluted in water and measured as described by Levy (1954) and Fraenkel-Conrat, Harris \& Levy (1955). An equally satisfactory solvent was butan-2-ol saturated with the phthalate buffer, which required only 15-20 hr. to run. The phosphate was used as above for the second run (see Fig. 1). These alcoholic solvents were found to be more convenient than the 'toluene-ammonia' solvent advocated by Fraenkel-Conrat et al. (1955), because no time was necessary for equilibration and because the solvent did not deteriorate. The 'toluene-ammonia' solvent was also undesirable in a laboratory where ninhydrin-amino acid determinations were being carried out.

The corrections necessary for the quantitative measurement of the $N$-terminal groups have been estimated by (a) serial measurement of each end group in samples given different periods of hydrolysis and $(b)$ recovery experiments on the free DNP-amino acids hydrolysed in the presence of

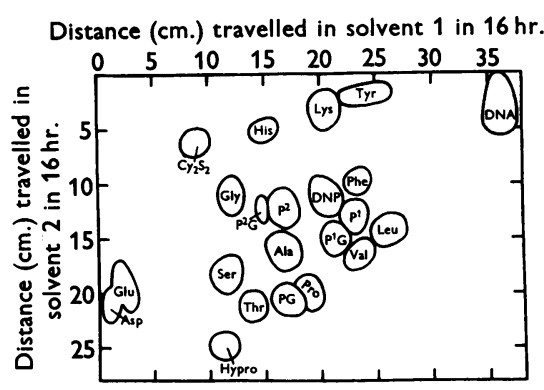

Fig. 1. Chromatography of DNP-amino acids and peptides on Whatman no. 7 paper buffered with $0.05 \mathrm{M}$-phthalate (pH 6). 0 is the starting point and both solvents were used descending the paper. Solvent 1, butan-2-ol shaken with excess of $0.05 \mathrm{M}$-phthalate buffer $(\mathrm{pH} 6)$, upper layer used. Solvent 2, 1.5 M-sodium phosphate buffer ( $\mathrm{pH} 6)$. Symbols for the DNP-amino acids, etc.: Asp, aspartic acid. Ala, alanine. $\mathrm{Cy}_{2} \mathrm{~S}_{2}$, cystine (bis). DNA, 2:4 dinitroaniline. DNP, 2:4-dinitrophenol (spot is bleached by acid vapour). Glu, glutamic acid. Gly, glycine, His, histidine (bis). Leu, leucine and isoleucine. Lys, lysine (bis). Hypro, hydroxyproline. Pro, proline. $\mathrm{P}^{\mathbf{1}}, \delta$ chloro- $\alpha$-DNP-aminovaleric acid. $\mathbf{P}^{2}, \alpha$-chloro- $\delta$-DNPaminovaleric acid. PG, prolylglycine [Hypro, Pro, PG and (more slowly) His, all darken in light]. $\mathrm{P}^{\mathbf{1}} \mathrm{G}, \delta$-chloro$\alpha$-DNP-aminovalerylglycine (DNP-methionine also runs here). $\quad P^{2} G$, probably $\alpha$-chloro- $\delta$-DNP-aminovalerylglycine. Phe, phenylalanine. Ser, serine ( $\epsilon$-DNP-lysine also runs here). Thr, threonine. Tyr, tyrosine (bis). Val, valine. With 2-methylbutan-2-ol-phthalate buffer as solvent 1 , the distribution of the derivatives is very similar, though the gap between the glutamic acid and serine is reduced. 
unlabelled histones. In $(a)$, in $11 \mathrm{~N}-\mathrm{HCl}$ at $100^{\circ}$, the values for each end group rose to a maximum at 3-5 hr. hydrolysis and then fell. Extrapolation to zero time gave an initial value but required correction for the manipulative losses and was subject to the uncertainty that the rate of destruction of the DNP derivative in peptide form may have been different from the rate after its liberation. In (b), comparison of samples before and after hydrolysis indicated the manipulative losses in this method. These were 10-20\%, depending on the derivative, but an average value of $20 \%$ loss has been adopted for all the derivatives encountered here. Much greater percentage losses occur where the amount of a given DNP-amino acid being handled is below $0.02 \mu$ mole.

The overall recoveries used in calculating the yields of end groups were: DNP-alanine 70\%, bis-DNP-lysine $69 \%$, DNP-valine and DNP-leucines 76\%, DNP-aspartic and -glutamic acids 65\%, DNP-serine $57 \%$, DNP-glycine $41 \%$ and DNP-proline $33 \%$. These all refer to $4.5 \mathrm{hr}$. hydrolysis at $100^{\circ}$ in $11.1 \mathrm{~N}-\mathrm{HCl}$. The DNP-proline value was based on the recovery of small amounts of DNP-proline and relatively large amounts of its two degradation products, $\delta$-chloro$\alpha$-DNP-amino- and $\alpha$-chloro- $\delta$-DNP-amino-valeric acid (Scanes \& Tozer, 1956). Trial degradations of DNP-prolylglycine (prepared from a sample of L-prolylglycine kindly given by Professor J. S. Fruton) and of DNP-proline in the presence of histones have been carried out. With DNPproline the maximum yields of $\delta$-chloro- $\alpha$-DNP-amino- and $\alpha$-chloro- $\delta$-DNP-amino-valeric acid were obtained after 0.75 and $1.5 \mathrm{hr}$. respectively in $11 \mathrm{~N}-\mathrm{HCl}$ at $100^{\circ}$, whereas with DNP-prolylglycine the times were 3 and $5.5 \mathrm{hr}$. respectively, periods much closer to those observed in the hydrolysis of DNP-histones, in which the maximum yields of $\delta$-chloro- $\alpha$-DNP-amino- and $\alpha$-chloro- $\delta$-DNP-aminovaleric acid were reached after 2.5 and $6 \mathrm{hr}$. respectively.

In $4.5 \mathrm{hr}$. hydrolysis the total recovery of DNP-proline and $\delta$-chloro- $\alpha$-DNP-amino- and $\alpha$-chloro- $\delta$-DNP-aminovaleric acid from DNP-prolylglycine was $33 \%$, and this value has been adopted as the value to be used with the DNP-histones. It is of interest that in the degradation of DNP-prolylglycine up to 2 or $3 \mathrm{hr}$. duration, spots due to $\delta$-chloro- $\alpha$-DNP-amino- and probably $\alpha$-chloro- $\delta$-DNPamino-valerylglycine appear on the chromatograms as well as those of DNP-proline, 2:4-dinitrophenol and unchanged DNP-peptide. The positions of these substances as well as most of the common DNP-amino acids on the chromatograms are shown in Fig. 1.

The amount of DNP-proline itself on the chromatograms from DNP-histones is so small that it is unnecessary in the calculation of the yield of $N$-terminal proline to allow for the difference in molecular extinction coefficient between it and $\delta$-chloro- $\alpha$-DNP-amino- and $\alpha$-chloro- $\delta$-DNP-aminovaleric acid.

The acid-soluble part of the hydrolysate of DNP-histones was evaporated to dryness and run on Whatman no. 1 paper in butanol-acetic acid-water $(5: 1: 4$, by vol.). The $\epsilon$-ENPlysine formed a wide band which may also have contained $\alpha$-DNP-histidine and DNP-arginine. After eluting the band with $0 \cdot 1 \mathrm{~N}-\mathrm{HCl}$ chromatographically the dried extract was coupled with FDNB (Levy, 1954). Extraction of the acidified products with ether removed all the e-DNP-lysine as bis-DNP-lysine. No $\alpha$-DNP-histidine or DNP-arginine could be detected on chromatograms of the acid-soluble remainder and no bis-DNP-histidine appeared either in the ether- or acid-soluble products.
The other part of the acid-soluble material, running more slowly than the $\epsilon-D N P$-lysine band, was nearly colourless, though $N^{1(3)}$-DNP-histidine appeared as a grey band after exposure to light for some hours. All this slow-running material was eluted with $0 \cdot 1 \mathrm{~N}-\mathrm{HCl}$, dried and recoupled with FDNB. This was done to estimate (as bis-DNP-lysine) the proportion of free lysine in the hydrolysates of the DNPhistones, since if the original reaction with histones was incomplete free lysine would appear in the hydrolysate (assuming that lysine $\epsilon$-amino groups are not blocked by some steric effect or bonding). In three hydrolysates no bis-DNP-lysine appeared, whereas in a $6 \mathrm{~N}-\mathrm{HCl}$ hydrolysate $\left(24 \mathrm{hr}\right.$. at $\left.110^{\circ}\right)$ of DNP-histones (H 1) a weak spot appeared amounting to less than $1 \%$ of the total lysine of the histones.

The nitroprusside reaction on acid hydrolysates of unlabelled histones and the Ehrlich test on chromatograms of barium hydroxide hydrolysates were both so weak that neither cystine nor tryptophan could contribute significantly to the end groups. The published analyses of histones show that these two acids are virtually absent (Davison \& Butler, 1954; Crampton et al. 1955). The ether, acid and acetone washings of DNP-histones obtained during their preparation have been examined chromatographically. Only negligible traces of DNP-amino acids were detected.

The proportion of histones in the DNP-histones has been taken as $77 \%$, being based on the number of DNP groups which could theoretically substitute in the whole histone according to the amino acid analysis published by Crampton et al. (1955).

\section{RESULTS}

The $N$-terminal groups found in the unfractionated histones were: proline and alanine, with smaller amounts of serine, lysine, leucine or isoleucine, valine, aspartic and glutamic acids, glycine and threonine. The proportions of the end groups (expressed as percentages of the total end groups) and the total quantities obtained in the guanidinebicarbonate and ethanol-bicarbonate media are given in Table 1. By comparing the results from histones $H 1$ in each reaction medium, it can be seen from the weight of histone/mole of end group that the total yield is greater in the material prepared in guanidine-bicarbonate. The duration of coupling beyond about $6 \mathrm{hr}$. had little effect on the yield of end groups.

The values for serine and lysine end groups were found to vary considerably. It is thought that some of the earlier high values for serine were due to the omission of the acid washing of the ether-soluble end groups, which would result in traces of $\epsilon-\mathrm{DNP}$ lysine being measured as DNP-serine, since it runs in exactly the same position on the two-dimensional chromatograms. Nevertheless, the average yield (moles/g. of histone) of serine end groups from histone preparation $\mathrm{H} l$ was nearly four times as high when guanidine was present in the coupling medium (see Table 1), so that it would appear that in this case the ethanol-bicarbonate medium fails to allow 1-fluoro-2:4-dinitrobenzene to react completely with serine end groups. However, in the 
later preparations of histones such as $H 16$ and $H$ 17, even with the guanidine medium, the serine end groups have been as low as $5 \%$ and the lysine values down to $1 \%$ of the total.

An examination was also made of the fractions of the histones prepared by $(a)$ extraction of the original nucleoprotein with citric acid (Davison \& Butler, 1954) and (b) precipitation of the histones by sodium chloride-ammonia (Davison \& Shooter, 1956). $H 7 / 1$ and $H 7 / 2$ are subfractions of the histones extracted by citric acid, which were obtained by chromatography on carboxymethylcellulose by the method of Davison \& Shooter (1956). H $18 \mathrm{~A}$ and $\mathrm{H} 18 \mathrm{~B}$ are the sodium chlorideammonia precipitate and supernatant products respectively derived from $H$ 18, a mixture of histone preparations $H 16$ and $H 17$. The results are given in Table 2, together with those obtained from the proteins extracted from a thymus-gland brei by $0.25 \mathrm{~N}-\mathrm{HCl}$ (sample $\mathrm{H} \mathrm{19}$ ). This material includes much protein which is not histone. It can be seen that these various fractions gave results which differed markedly from the original histones described above.

Fractions $H$ 7/1 and $H$ 7/2 account for most of the histones extracted by citric acid (which are only approximately $2 \%$ of the total histones in the nucleoprotein), and these fractions also account for most of the valine end groups found in the whole unfractionated histones. In another preparation of the citric acid-extracted histones, the proportions of the end groups in a $6 \mathrm{~N}-\mathrm{HCl}$ hydrolysate (12 hr. at $110^{\circ}$ ) were: valine $39 \%$, alanine $19 \%$, lysine $15 \%$, others $27 \%$. DNP-proline and its products were completely destroyed under these conditions.

Three samples of DNP-histones (H l) have been hydrolysed under more vigorous conditions to liberate any valine which might be present as DNP. valyl-peptides (such as the DNP-valyl-e-DNPlysine reported by Luck et al. 1956). Some valine peptides are notoriously stable to acid hydrolysis (Synge, 1945; Porter \& Sanger, 1948). However, hydrolysis in $6 \mathrm{~N}-\mathrm{HCl}$ at $108^{\circ}$ for $8 \mathrm{hr}$., at $110^{\circ}$ for $48 \mathrm{hr}$. or at $120^{\circ}$ for $24 \mathrm{hr}$., though increasing the proportion of valine and leucine (or isoleucine) end groups, only raised them from about 2 to 5 or $6 \%$ of the total end groups. No sign of the peptide DNP. valyl- $\epsilon$-DNP-lysine was seen on any of these chromatograms, and only after very short hydrolyses, e.g. $1 \mathrm{hr}$. at $100^{\circ}$ in $11 \mathrm{~N}-\mathrm{HCl}$, did any spots other than those in the positions of the known DNP-amino acids appear. Stable peptides were also

Table 1. N-Terminal groups of unfractionated histones

The proportions of $N$-terminal groups are expressed as percentages of the total end groups found.

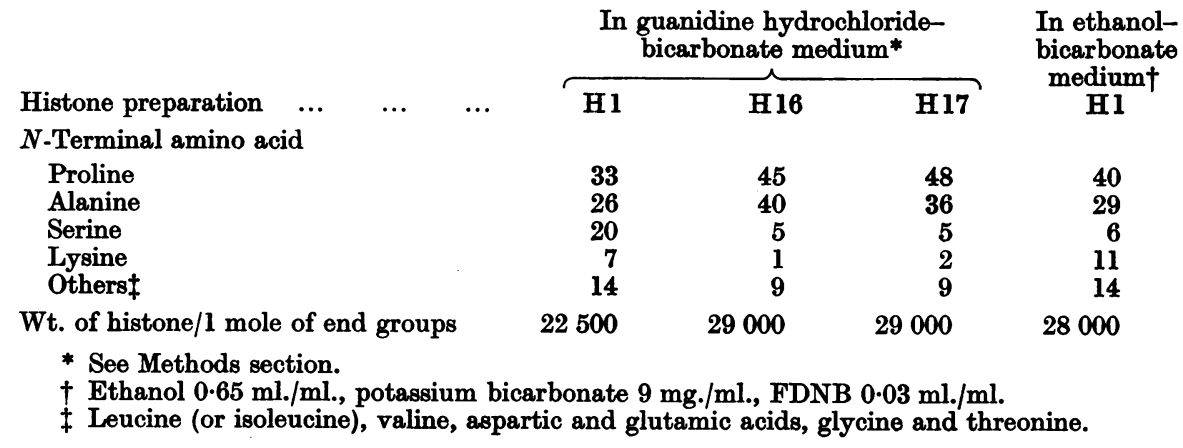

Table 2. N-Terminal groups of various histone fractions

The proportions of the $N$-terminal groups are expressed as percentages of the total end groups found. All were coupled with FDNB in the guanidine-bicarbonate medium.

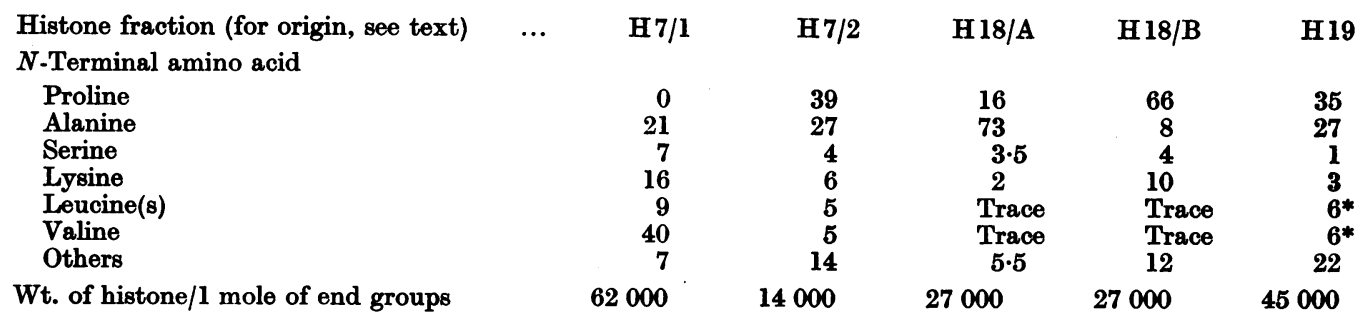

* From samples hydrolysed in $6 \mathrm{~N}-\mathrm{HCl}$ for $22 \mathrm{hr}$. at $110^{\circ}$. 
sought in the ethyl acetate extracts of the acidsoluble part of many of the hydrolysates of DNP. histones, but without success. Under the usual conditions of hydrolysis $\left(4.5 \mathrm{hr}\right.$. at $100^{\circ}$ in $\left.11 \mathrm{~N}-\mathrm{HCl}\right)$ only one spot appeared on chromatograms of such extracts, in the same position as DNP-serine. However, this spot was ninhydrin-positive and proved to be $\epsilon$-DNP-lysine.

\section{DISCUSSION}

The $N$-terminal groups of the thymus histones found above indicate the presence of two major types of peptide chain and about eight other types present in minor or trace amounts. The average chain weight is about 25000 . This value and the nature of the chief end groups are, with the exception of alanine, at variance with the results of Haley (1955) and Luck et al. (1956), which were obtained with histones prepared by extraction with sodium chloride and hydrochloric acid of thymuscell nuclei. In whole-thymus histones these authors found that valine accounted for $53 \%$ of the $N$. terminal residues, leucine for $21 \%$, alanine for $18 \%$ and glycine for $8 \%$. The value of $1 \mathrm{~g}$. mol. of end groups/8000 $\mathrm{g}$. of histones was supported by their sedimentation and osmotic-pressure studies.

It is difficult to account for these differences except on the assumption that degradation of the histones occurred in the preparative method used by Luck et al. (1956). Although the histones are known to aggregate in alkaline solution (Davison et al. 1954), the reaction with 1-fluoro-2:4-dinitrobenzene is thought to have been complete since virtually no free lysine could be detected in the hydrolysates of DNP-histones. The hydrolyses are thought to have liberated most of the end groups since more severe conditions did not raise the values of terminal DNP-valine and DNP-leucine (which can give very stable DNP-peptides) to values anywhere near those of DNP-proline or DNP-alanine. The corrections for hydrolytic and manipulative losses are thought to have been adequate, though in some work with DNP-proteins, e.g. with hypertensin (Elliott \& Peart, 1957) and with corticotrophin (Levy, Geschwind \& Li, 1955), the losses of terminal DNP-amino acids were greater than expected. Finally, the washings from the preparations of the DNP-histones have revealed no loss of terminal groups such as Thompson (1953) encountered in the study of carboxypeptidase.

Experiments made with the object of causing slight degradation of the histones, by keeping a solution at $\mathrm{pH} 7$ for 2 days or in $0.1 \mathrm{~N}$-sodium hydroxide for a day, or by heating the aqueous solution at $100^{\circ}$ for an hour, caused only very slight increases in some of the end groups. Another experiment wherein all the acid-soluble protein (not merely the histones) from thymus glands was coupled, after dialysis, with 1-fluoro-2:4-dinitrobenzene showed that the amounts of valine and leucine end groups (each was $6 \%$ of the total) were small compared with the proline and alanine end groups, which were 35 and $27 \%$ of the total end groups respectively.

The sodium chloride-ammonia fractionation of the histones has been shown to precipitate the histones which are relatively richer in arginine and migrate faster on electrophoresis, and to leave in the solution the histones which are relatively richer in lysine and which migrate more slowly on electrophoresis (Davison \& Shooter, 1956). Table 2 shows further that most of the arginine-rich histones (H 18A) have alanine $N$-terminal groups and most of the lysine-rich ones (H 18B) have proline $N$ terminal groups. The small amounts of histones with other end groups fall into both of these main classes.

The origin of the differences in yield of serine and lysine end groups in different preparations of the mixed histones has not been established. In the later preparations both of these acids formed only a minor proportion of the $N$-terminal groups. There may be variations in the proportions of the different histones obtained from different animals or breeds of cattle. Allfrey, Mirsky \& Osawa (1955) have noted the variability in properties of thymus nuclei prepared at different times of the year. Moreover, in the preparative stages involving washings and dialyses near neutrality there is the possibility of enzyme action causing slight degradation of peptide chains.

\section{SUMMARY}

1. The chief $N$-terminal groups of the histones extracted from calf-thymus glands are proline and alanine, with smaller amounts of lysine and serine, and still less of six other amino acids.

2. The proportions of the serine and lysine end groups have declined markedly in later preparations of the histones.

3. The quantities of the end groups gave an average weight of about $25000 / \mathrm{g}$. mol. of end groups.

4. In a fractionation of the histones, those precipitated by sodium chloride-ammonia (the arginine-rich histones) mostly have alanine $N$-terminal groups, whereas those remaining in solution in sodium chloride-ammonia (the lysine-rich histones) mostly have proline end groups.

I wish to thank Professor J. A. V. Butler, F.R.S., for his continual encouragement and Miss P. Simson and Mr E. W. Johns for nitrogen analyses and histone preparations respectively. This investigation has been supported by grants to the Chester Beatty Research Institute (Institute of Cancer Research, Royal Cancer Hospital) from the 
British Empire Cancer Campaign, the Jane Coffin Childs Memorial Fund for Medical Research, the Anna Fuller Fund and the National Cancer Institute of the National Institutes of Health, U.S. Public Health Service.

\section{REFERENCES}

Allfrey, V. G., Mirsky, A. E. \& Osawa, S. (1955). Nature, Lond., 176, 1042.

Blackburn, S. \& Lowther, A. G. (1951). Biochem. J. 48, 126. Crampton, C. F., Moore, S. \& Stein, W. H. (1955). J. biol. Chem. 215, 787.

Daly, M. M. \& Mirsky, A. E. (1955). J. gen. Physiol. 38, 405.

Dasler, W. \& Bauer, C. D. (1946). Industr. Engng Chem. (Anal.), 18, 52.

Davison, P. F. \& Butler, J. A. V. (1954). Biochim. biophys. Acta, 15, 439.

Davison, P. F., James, D. W. F., Shooter, K. V. \& Butler, J. A. V. (1954). Biochim. biophys. Acta, 15, 415.
Davison, P. F. \& Shooter, K. V. (1956). Bull. Soc. chim. Belg. 65, 85.

Elliott, D. F. \& Peart, W. S. (1957). Biochem. J. 65, 247.

Fraenkel-Conrat, H., Harris, J. I. \& Levy, A. L. (1955). In Methods of Biochemical Analysis, vol. 2, p. 363. Ed. by Glick, D. New York: Interscience Publishers Inc.

Haley, M. I. (1955). Diss. Abstr. 15, 962.

Levy, A. L. (1954). Nature, Lond., 174, 126.

Levy, A. L., Geschwind, I. I. \& Li, C. H. (1955). J. biol. Chem. 213, 187.

Luck, J. M., Cook, H. A., Eldredge, N. T., Haley, M. I., Kupke, D. W. \& Rasmussen, P. S. (1956). Arch. Biochem. 65, 449.

Mills, G. L. (1952). Biochem. J. 50, 707.

Porter, R. R. \& Sanger, F. (1948). Biochem. J. 42, 287.

Sanger, F. (1945). Biochem. J. 39, 507.

Scanes, F. S. \& Tozer, B. T. (1956). Biochem. J. 63, 282.

Stedman, E. \& Stedman, E. (1951). Phil. Trans. 235, 565.

Synge, R. L. M. (1945). Biochem. J. 39, 351.

Thompson, E. O. P. (1953). Biochim. biophys. Acta, 10, 633.

\title{
Studies on the Biosynthesis of Riboflavin
}

\section{PURINE METABOLISM AND RIBOFLAVIN SYNTHESIS IN EREMOTHECIUM ASHBYII*}

\author{
By E. G. BROWN, T. W. GOODWIN AND O. T. G. JONES \\ Department of Biochemistry, The University, Liverpool 3
}

\section{(Received 15 May 1957)}

It is now well established that purines stimulate the biosynthesis of riboflavin in the fungus Eremothecium ashbyii (MacLaren, 1952; Goodwin \& Pendlington, 1954), and experiments with isotopically labelled adenine indicate that this is due to the direct incorporation of purine after loss of $\mathrm{C}_{(8)}$ (McNutt, 1954).<smiles>c1ncc2[nH]cnc2n1</smiles><smiles>Cc1cc2nc3c(=O)[nH]c(=O)nc-3n(CC(O)CO)c2cc1C</smiles>

Purine

Riboflavin
Experiments with labelled glycine, formate and serine also indicate that rings $B$ and $C$ of riboflavin are built up in a very similar pattern to the purine rings (Plaut, 1953; 1954 $a, b$; Goodwin \& Jones, 1956). Naturally occurring pyrimidines do not stimulate flavinogenesis (Brown, Goodwin \& Pendlington, 1955).

Goodwin \& Pendlington (1954) found that xanthine and adenine both stimulated flavino-

* Part 3: Goodwin \& Jones (1956). genesis and that xanthine was the more efficient. As xanthine is structurally the purine most closely related to riboflavin, it is likely that other purines are active by virtue of their conversion into xanthine. One would expect that the first step in the conversion of adenine would be the formation of hypoxanthine, for an adenase (adenine deaminase) has been observed in a number of micro-organisms, especially fungi (Di Carlo, Schultz \& McManus, 1951; Roush, 1954). The hypoxanthine produced would then be converted into xanthine by xanthine oxidase. Preliminary experiments showed, however, that hypoxanthine is much less active than adenine and thus would appear to be ruled out as an intermediate.

Because of these somewhat unexpected results it was decided $(a)$ to extend the number of purines investigated to include hypoxanthine, uric acid and guanine, the ribosides adenosine and inosine, and the ribotide adenosine-3'-phosphate; $(b)$ to follow the metabolism of these compounds during their incorporation into riboflavin, and (c) to examine the effect on riboflavin synthesis of certain purine antagonists and inhibitors of purine synthesis.

A brief report of some of these findings has already appeared (Brown, Goodwin \& Jones, 1956). 